\title{
THE EFFECT OF METFORMIN ON BODY WEIGHT IN POLYCYSTIC OVARY SYNDROME PATIENTS
}

Yasser El-Shabrawy*1, Alaa Alhaboby ${ }^{2}$, Nageeb Abdulgaleel Hassan ${ }^{3}$, Sameer Issa Bloukh ${ }^{4}$ and Moayad Jamal Shahwan ${ }^{5}$

$* 1,2,3,4,5$ College of Pharmacy and Health Sciences, Ajman University of Science and Technology, P.O.Box 346 Ajman, UNITED ARAB EMIRATES

*Correspondence Author: moyadshahwan@yahoo.com

Abstract:

Polycystic Ovary Syndrome (PCO) is an endocrine disease that is characterized by a set of symptoms including irregular menstruation, subfertility, skin changes, hirsutism and weight gain.

The pathogenesis of $\mathrm{PCO}$ is mostly related to increase androgens and insulin resistance. Treatment is mostly symptomatic hence metformin is being used due to its effect in decreasing gluconeogenesis and by increasing peripheral utilization of glucose, in addition to its lowering effects on androgen. Thus, Metformin role in PCO treatment ranges between managing insulin resistance, promoting ovulation and treating hirsutism to some extent. Some studies showed that Metformin is effective in lowering body weight in PCO patients, yet it is not being indicated officially for this purpose.

Background: Metformin is expected to have an effect of weight reduction in PCO patients This is the scope of this research which intends to explore this action.

Method: The Study design is cross sectional study using online questionnaire. Open and closed ended questions were included.

Results: Based on the results the majority shows decrease in the weight, while only one reported increase in their weight and only one had the same weight during and after treatment with metformin.

Conclusions: Metformin generally showed positive effect regarding reducing the body weight of the females diagnosed with polycystic ovarian syndrome when used alone.

Keywords:

Polycystic Ovary Syndrome, metformin, diabetic, insulin resistance, body mass index.

\section{INTRODUCTION}

Polycystic ovary syndrome (PCO) is one of the most common endocrine disorders [1]. It affects $5-10 \%$ of women in the reproductive age group [2,3]. Since it is a syndrome, it is difficult to define it and several definitions were attempted [4]. A syndrome is a combination of a set of symptoms that usually appear in one condition [5]. PCOs remains a syndrome and a such no single diagnostic criterion is sufficient for clinical diagnosis [6].

Although PCOS is not completely reversible, there are a number of treatments that can reduce or minimize some symptoms. Most women with PCOS are able to lead a normal life without 


\section{INTERNATIONAL JOURNAL of RESEARCH -GRANTHAALAYAH \\ A knowledge Repository}

Science

significant complications. [7]. If the case was elevated androgen levels, it can sometimes cause excessive facial hair growth, acne, and/or male-pattern hair thinning. Most, but not all, women with PCOS are overweight or obese, and they are at higher than average risk of developing diabetes and obstructive sleep apnea [6]. One of the medications given in such cases is metformin.

Metformin $\left(\mathrm{C}_{4} \mathrm{H}_{11} \mathrm{~N}_{5}\right.$, ) is an oral antidiabetic drug, it is a the only available medication in the Biguanides class that is used in this case. It is especially used for type 2 diabetes, because it increases responsiveness of the body to insulin. Metformin effect is mainly due to decreasing gluconeogenesis and by increasing peripheral utilization of glucose [8]. Due to this mechanism, metformin is being used in polycystic ovary syndrome patients as it decreases the insulin resistance noticed in such cases.

In addition to this, it has been found that metformin decreases the level of androgens produced by the ovaries and adrenal glands. These two actions helps regulate menstrual cycles, start ovulation, and lower the risk of miscarriage in women with PCOS. Long-term use also lowers diabetes and heart disease risk related to high insulin levels. [2,9]

Metformin is also expected to have an effect of weight reduction in PCO patients [10]. This is the scope of this research which intends to explore this action.

\section{MATERIAL AND METHODS}

Based on the importance of polycystic ovarian syndrome and how it affects the patients' lives, survey was made and distributed randomly.

Study design is cross sectional study using online questionnaire. Open and closed ended questions were included.

The population sample is random; the questionnaire was for all eligible females. Inclusion criteria are female gender who can access and fill online questionnaires from any nationality. Exclusion criteria include male gender and children.

The sample size depended on the number of respondents within the time frame of this study, which is one month. Hence the sample size at the end of the month was 223 females. Data was analysed using Microsoft Excel.

\section{ETHICAL ISSUES}

The information about the study was fully explained and consent was taken before starting the questionnaire. Patients were not asked about their name or any personal information that are not related to the research. All other information were confidential. 


\section{INTERNATIONAL JOURNAL of

\section{RESULTS AND DISCUSSIONS}

Exploring the relationship between metformin and weight loss in polycystic ovarian syndrome patients

Age

More than $50 \%$ were between 20 and 30 years old.

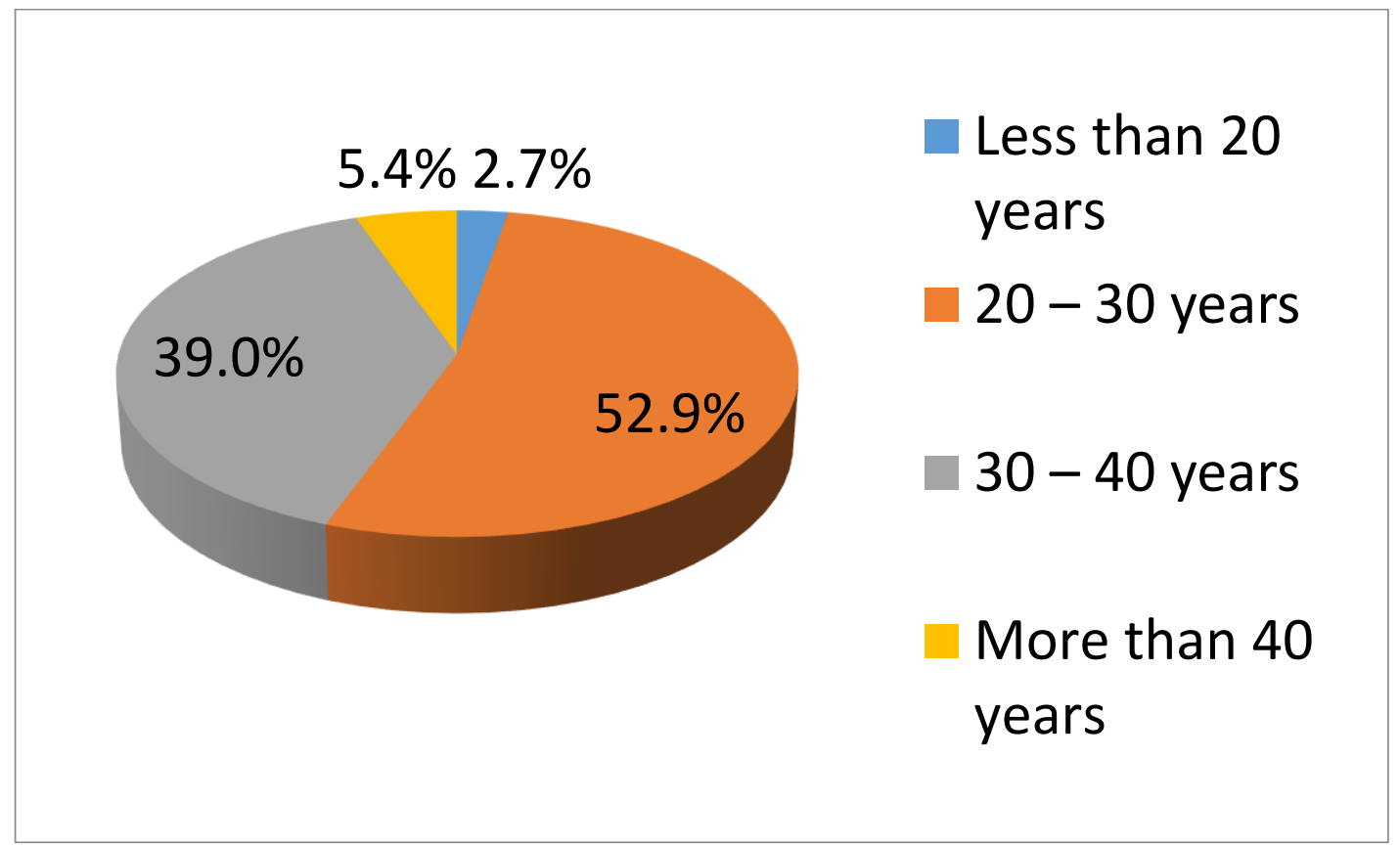

Figure 1 Age distribution

Most females who responded to the survey were between 20-30 years old as shown in the results (52\%) followed by $39 \%$ of $30-40$ years old, then $5.4 \%$ more than 40 and the minimum percentage was $2.7 \%$ for age less than 20 . This maybe an indicator that polycystic ovarian syndrome occur mostly in the most reproductive age which is 20-30 years old, and the chances to develop it decrease with increasing age, this result is supported by previously mentioned studies which states that about $5-10 \%$ of women of reproductive age are diagnosed with PCO [2, 3].

\section{$\underline{\text { Age of diagnosed females }}$}

$57 \%$ of diagnosed females with polycystic ovarian syndrome age were between 20 to 30 years old. 

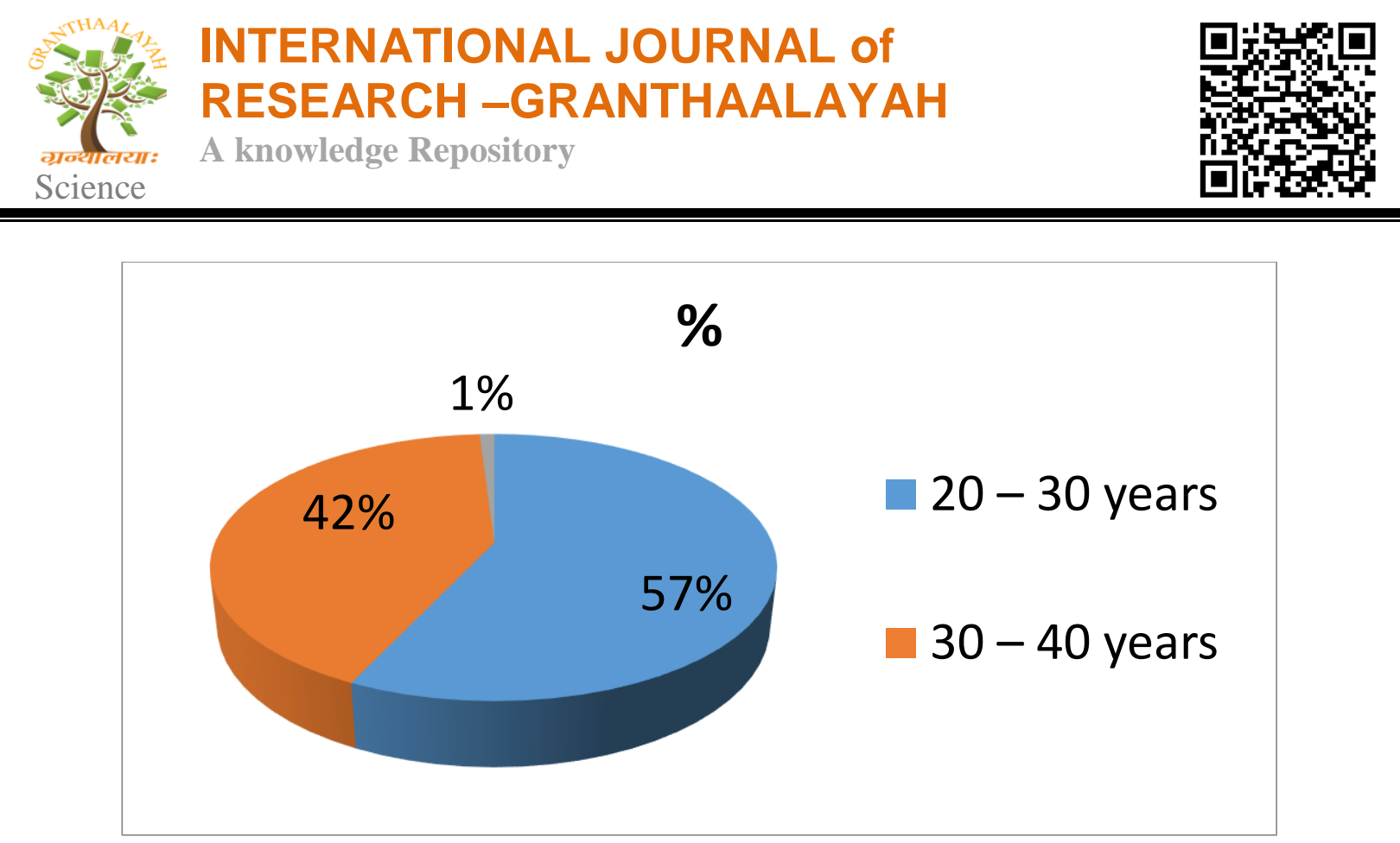

Figure2 Age of diagnosed females

According to the results, the highest number of females who were diagnosed with polycystic ovarian syndrome were between 20 and 30 years old (57\%), followed by $42 \%$ were between 30 to 40 years old and only $1 \%$ were above 40 and none less than 20 .

This maybe an indicator that polycystic ovarian syndrome occur mostly in the most reproductive age which is 20-30 years old, and the chances to develop it decrease with increasing age.

\section{$\underline{\text { Height }(\mathrm{cm}) \text { and Weight }(\mathrm{kg})}$}

BMI (body mass index) was calculated and around 50\% are overweight and obese.

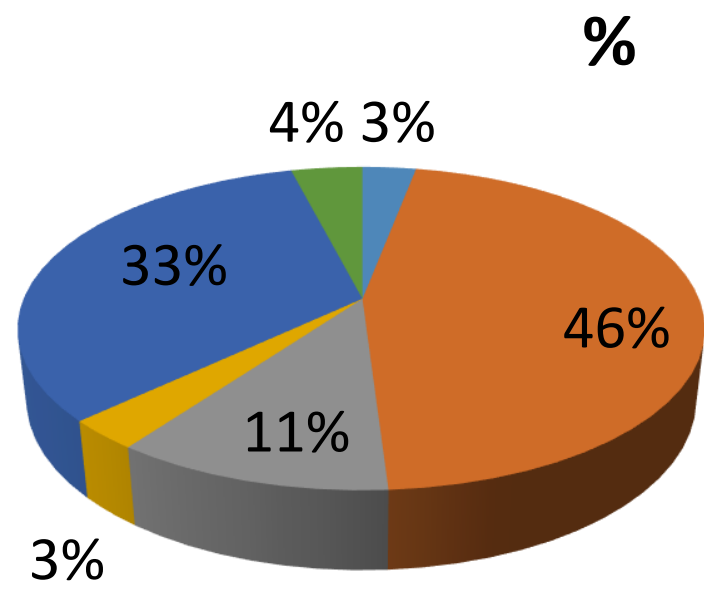

Morbid obesity

Normal weight

Obesity I

Obesity II

Overweight

Figure 3 Height and weight 


\section{INTERNATIONAL JOURNAL of RESEARCH -GRANTHAALAYAH \\ A knowledge Repository}

Science

Of all females whom were diagnosed and non-diagnosed with polycystic ovarian syndrome the BMI was calculated and $46 \%$ had normal weight, 33\% were overweight, $11 \%$ had obesity 1, 3\% had obesity 2 and $3 \%$ were morbid obese, and lastly $4 \%$ were underweight.

Overall around 50\% are obese and overweight which may highlight that awareness must be increased regarding this matter as obesity is risk factor for many serious diseases.

\section{BMI for diagnosed females}

Around $60 \%$ are overweight and obese.

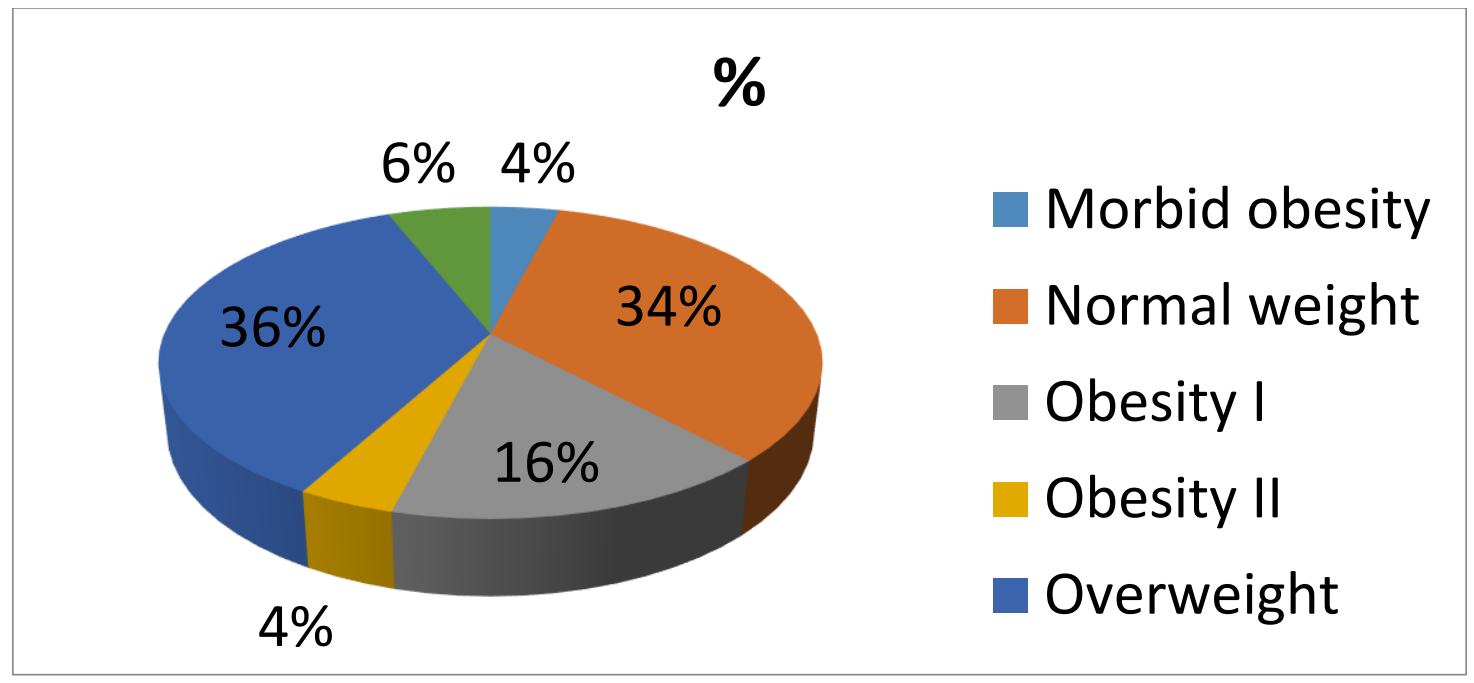

Figure 4 BMI for diagnosed females

The body mass index (BMI) of diagnosed females were calculated and the results showed that $36 \%$ of them were overweight, $34 \%$ had normal weight, $16 \%$ had obesity $1,4 \%$ obese stage $2,4 \%$ morbid obese and $6 \%$ were underweight.

So about $60 \%$ had weight above their ideal weight and the cause is probably due to insulin resistance that is accompanied with the polycystic ovarian syndrome. Results showed that most of them were overweight and obese which supports the studies that stated obesity as one of the major symptoms of polycystic ovarian syndrome, confirmatory to this result is the previously mentioned study which found that about $44 \%$ of women with polycystic ovaries are obese. [4] [11] Another previously mentioned study stated that about $40-45 \%$ of diagnosed women with polycystic ovary show obesity. [12] 


\section{INTERNATIONAL JOURNAL of RESEARCH -GRANTHAALAYAH

For the females who reported weight changes after treatment (Metformin only)

Most of patients lost weight.

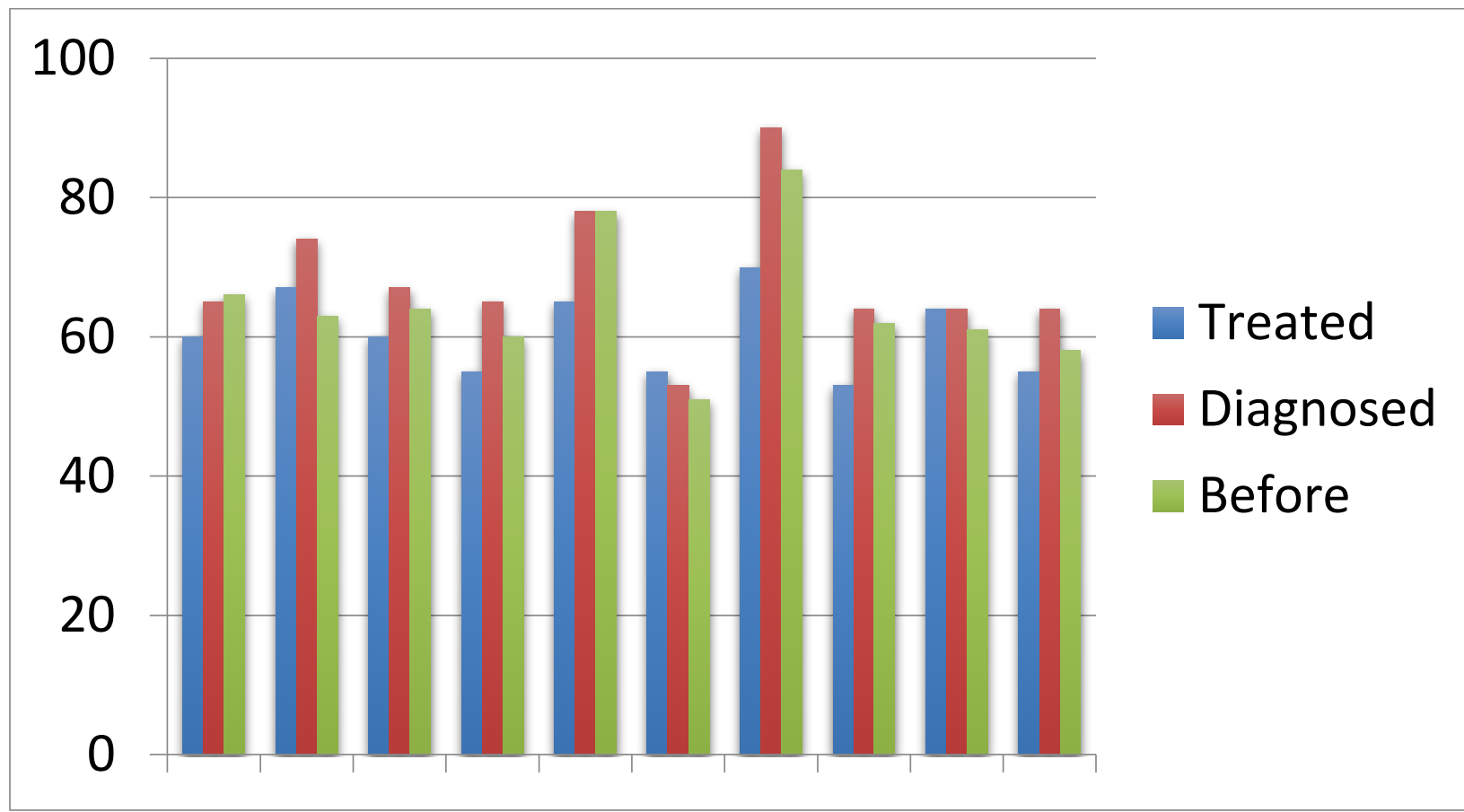

Figure 5 Reported weight changes after taking metformin only

This graph shows the weight of females before and during diagnosis and after starting metformin, majority shows decrease in the weight, while only one reported increase in their weight and only one had the same weight during and after treatment.

The reason is probably that these two that had no change and increase in their weight that they just started the treatment, or are not eating healthy diet as recommended by most studies.

Results showed that $80 \%$ of diagnosed women are taking metformin and this might be a good indicator that doctors prefer to prescribe this medication and patients accept to use it even though most of them are not diabetic (considering metformin is mainly used for type 2 diabetes [8] ), comparing this result with previous studies it was found that metformin was proved to have beneficial effects on patients especially regarding their weight. As one previously mentioned study stated that Metformin had positive effect on reducing body weight[13], another study proved that using metformin along with diet and exercise had significant positive effect[14],

Yet the other $20 \%$ are not taking metformin and the reason may be they are sensitive to it due to its side effects or that they are suffering from renal or liver disease and in that case metformin is contraindicated 


\section{INTERNATIONAL JOURNAL of RESEARCH -GRANTHAALAYAH

Science

\section{CONCLUSION AND RECOMMENDATIONS}

Metformin generally showed positive effect regarding reducing the body weight of the females diagnosed with polycystic ovarian syndrome when used alone,

Recommendations: Metformin showed beneficial effect alone, but more investigations needed regarding combining it with other kind of PCO treatments such as oral contraceptives or clomiphene.

\section{REFERENCES}

[1]. Mathur, R., et al., Use of metformin in polycystic ovary syndrome. American journal of obstetrics and gynecology, 2008. 199(6): p. 596-609.

[2]. Franks, S., Polycystic ovary syndrome. New England Journal of Medicine, 1995. 333(13): p. 853-861.

[3]. Atiomo, W. and A. Sinha, The role of metformin in the treatment of infertile women with polycystic ovary syndrome. The Obstetrician \& Gynaecologist, 2004. 6(3): p. 145-151.

[4]. Carmina, E. and R.A. Lobo, Polycystic ovary syndrome (PCOS): arguably the most common endocrinopathy is associated with significant morbidity in women. Journal of Clinical Endocrinology \& Metabolism, 1999. 84(6): p. 1897-1899.

[5]. Lorig, K.R., et al., Internet-based chronic disease self-management: a randomized trial. Medical care, 2006. 44(11): p. 964-971.

[6]. Baker, P.N. and L. Kenny, Obstetrics by ten teachers. 2011: CRC Press.

[7]. Polycytic ovary syndrome. 2014 30-4-2014]; Available from: http://www.uptodate.com/contents/polycystic-ovary-syndrome-pcos-beyond-the-basics.

[8]. BNF, British national formulary. Vol. 65. 2013: Pharmaceutical Press.

[9]. Ehrmann, D.A., Polycystic ovary syndrome. New England Journal of Medicine, 2005. 352(12): p. 1223-1236.

[10]. Harborne, L.R., et al., Metformin and weight loss in obese women with polycystic ovary syndrome: comparison of doses. Journal of Clinical Endocrinology \& Metabolism, 2005. 90(8): p. 4593-4598.

[11]. De Groot, P., et al., PCOS, coronary heart disease, stroke and the influence of obesity: a systematic review and meta-analysis. Human reproduction update, 2011. 17(4): p. 495-500.

[12]. Glintborg, D., et al., Body composition is improved during 12 months treatment with metformin alone or combined with oral contraceptives compared to treatment with oral contraceptives in polycystic ovary syndrome. The Journal of Clinical Endocrinology \& Metabolism, 2014.

[13]. Dunaif, A., Insulin action in the polycystic ovary syndrome. Endocrinology and metabolism clinics of North America, 1999. 28(2): p. 341-359.

[14]. Diamanti-Kandarakis, E., Insulin sensitizers targeting metabolic and reproductive consequences in polycystic ovary syndrome, in Polycystic Ovary Syndrome. 2008, Springer. p. 197-215. 